\title{
Using Laboratory to Improve Understanding of 802.3 Physical Characteristics
}

\author{
Jaime Benjumea, Ana V. Medina, Sergio Martín, Gemma Sánchez \\ Departamento de Tecnología Electrónica, University of Seville, Spain \\ benjumea@dte.us.es
}

\begin{abstract}
When teaching computer networks as part of a Computer Engineering degree, emphasis is placed on higher-layer protocols while Physical and Data-Link layers usually play a secondary role. Physical aspects of data communication are sometimes taught as an independent, not-related, topic whereas Data-Link concepts are "hard" to our students. We propose a new lab experience in which students can see IEEE 802.3 frames in a new way; by using standard digital oscilloscope we are able to go deeper into lower-level layers. The lab session will be divided into two parts, focusing on topics that are usually considered "hard" by our students. The first part will focus on physical aspects of a frame transmission whereas the second will focus on MAC-layer. For example, topics like physical layer synchronization, signal attenuation, 5-4-3 rule and even Interframe Gap are exercised at the lab session. This experience has been tested with a group of last year students; we have analyzed students "own perception" of previous knowledge of some related topics, the results from a evaluation questionnaire (a small exam given out to students) and students evaluation of this exercise.
\end{abstract}

Index Terms - computer engineering education, constructivism learning, IEEE 802.3 standard, laboratory, MAC layer, physical layer.

\section{INTRODUCTION}

Arquitectura de Redes de Computadores (Computer Networks Architecture) in Ingeniero en Informática (a fiveyear Computer Engineering degree taught at University of Seville) is taught within the fourth year. As this is the first time students receive a specific formation in Computer Networks, students have no previous knowledge in signal theory and, as a result, no knowledge in the physical aspects involved in communication between computers.

Due to lack of time, no other subject in the degree deals with physical-layer so these concepts are slighted introduced in Arquitectura de Redes de Computadores. So topics as clock synchronization, digital or analog codification, attenuation, propagation delay, bauds or bits per second are commonly used in classroom. Experience has shown that these topics are usually tricky for the students because these topics are, somehow, quite theoretical; this means that a concept like, for example, clock synchronization is not properly understood even though it is a very simple concept. The same problem is observed when teaching certain MAClayer topics of the IEEE 802.3 LAN protocol. It is worth to mention that there is no lack of bibliography related with these topics, on the contrary [1][2][3] are only some examples of bibliography covering them.

Consequently, it seems convenient to complement lectures with practical laboratory exercises allowing students to understand better concepts related to physical-layer characteristics. In addition, MAC-layer related concepts might also be added to practical exercises because, even though some of them (like frame format) can be shown using software (network analyzer programs), others (transmission order, Inter Frame Gap, etc) can only be shown using a digital oscilloscope.

This paper is divided as follows. Section II justifies the use of IEEE802.3 technology as a basis for laboratory exercises. Section III describes problematic topics and details why these topics are especially difficult for students. Section IV describes the methodology to be used in the exercise proposed. Section V describes the laboratory requirements. In section VI a sample of questions made to students during practical session is given; in this case, the expected answers from students are outlined. In section VII a hands-on experience is described (with an evaluation from the students). Final conclusions are given in section VIII.

\section{Choosing an Adequate Lab Scenario}

Using a standard wave generator and an oscilloscope, would allow introducing physical related topics in a practical exercise in any electronics laboratory. However, as students expect to have practical exercises directly related to networking world, making them to work with a wave generator is probably not a good idea.

On the other hand, the waveform generated from an IEEE802.3 LAN at $10 \mathrm{Mbps}$ is simple enough to be captured on a digital oscilloscope; so using both the IEEE802.3 LAN technology (working at $10 \mathrm{Mbps}$ ) as "wave generator", standard LAN equipment and a digital oscilloscope makes it possible not only to analyze certain physical topics but also allows to analyze some IEEE802.3 MAC-layer topics; this means that both layers, physical and MAC, could be covered in a single practical exercise.

However, only $10 \mathrm{Mbps}$ alternative within this protocol is appropriate in order to fulfill the objectives described 
Session M2E

before; there are other alternatives in twisted pair, but they are not appropriate because speeds above $10 \mathrm{Mbps}$ use more complicated codifications (e.g. 100Mbps uses 4B/5B) which would increase exercise difficulty. Also, material available in laboratory does not allow capturing the waveform generated at $100 \mathrm{Mbps}$ or faster speeds. In any case, using the low speed alternative should not be a problem as the objective is to improve the understanding of certain IEEE802.3 parameters which are the same, regardless of the network speed.

\section{TOPICS TO BE COVERED}

Next step is to specify which topics will be covered in the practical exercise proposed. The suggested topics have been selected from a series of themes, based on previous experience, that are problematic for students. Topics will be classified into two groups: Physical-related and MACrelated; for every topic, there is a brief description of the problem.

\section{A. Physical-related topics}

- Attenuation: Signal attenuation is a simple concept that can be easily understood. However, students sometimes think that signal looses power in a linear way, so they tend to think that if a signal looses $1 \mathrm{~V}$ in $5 \mathrm{~m}$, it will lose $2 \mathrm{~V}$ in $10 \mathrm{~m}$.

- Propagation delay (medium): This concept is sometimes mixed-up with transmission time (i.e. some kind of confusion is created between bits-per-second and meters-per-second). Allowing students to measure these delays will hopefully help them to clarify both concepts and realize that propagation delay might be, in some cases, appreciable.

- Propagation delay (devices): Hubs are used in a LAN because signal decays with distance, this is a simple concept that students usually understand well. But it is not so evident that signal regeneration takes time; how much time is one of the topics students will be able to test at laboratory. By measuring it, students should notice that hub induced delay is significant and thus, infer that its number must be limited in order to comply with 5-4-3 rule (this concept is studied in MAC-related topics section).

- Synchronous transmission: IEEE802.3 protocol, when operating at $10 \mathrm{Mbps}$, uses a Manchester encoding; this is a good example for students to learn why preamble in a frame is coded as 7 bytes '10101010'. Exercises at laboratory might help students to understand why preamble is so large, and why this stream of bits helps the receiving side to synchronize its clock (so there is no need of an external clock).

\section{B. MAC-related topics}

- 5-4-3 rule: Carrier Sense Multiple Access / Collision Detection (CSMA/CD) protocol is used in IEEE802.3 networks when half-duplex mode is in use. As $\mathrm{CSMA} / \mathrm{CD}$ expects to detect collisions (i.e. simultaneous transmission from two or more nodes) before frame transmission is over, there is a limit in the number of segments and hubs within a single collision domain. Students usually misinterpret this limit, often known as 5-4-3 rule, as they fail to understand that this limit (as well as minimum frame length) is a CSMA/CD constrain and not a physical constrain. It is also important to understand that 5-4-3 rule (after all, a way to limit Round Trip Delay) and minimum frame length are topics that link together, so changing one will lead to changes in the other.

- Transmission order: IEEE802.3 standard states that bytes within a frame are transmitted in order but least significant bit is transmitted first. However, in most computers, bits within a byte are represented just in the opposite order, NICs manufacturers also use the latter notation. So there are two notations (for the same MAC address): canonical (notation used by NICs manufactures) and transmission. It is important that students understand the differences between them and, consequently, interpret properly individual/ Group bits in a MAC address..

- Minimum frame size: IEEE802.3 imposes a minimum frame length so any transmitted frame will always be equal or larger than this size (512 bits, excl. preamble and SFD). Even though no special difficulties are present when understanding this concept, it is useful that they can see by themselves that any frame transmitted has, at least, this size and that they can obtain its size experimentally, by using oscilloscope cursors.

- Start Frame Delimiter (SFD): This field, along with the preamble, is not shown in software network analyzers. Students will be able to see how the SFD is used to indicate the beginning of a frame.

\section{Other topics}

There are some advanced topics that might be introduced in a practical exercise. Although they are not part of the original exercise, the following topics can also be proposed:

- Inter Frame Gap (IFG): This gap must last a minimum of 96 bits, that way the minimum distance between two consecutive frames is always 9,6 microseconds when operating at $10 \mathrm{Mbps}$. The IFG imposes a period of silence between two consecutive frames that keeps a station away from saturating the medium and allows the receiver to determine the end of a frame.

- Different commutation modes: When using switches in a LAN, there are two different commutation modes: store and forward, and fast forwarding. Both modes can be observed and compared at the laboratory.

\section{Proposed Methodology}

It is crucial that students work on a constructivism basis, so they will always work on topics previously studied by them

October 18 - 21, 2009, San Antonio, TX 
(either individually and/or by lectures held prior to laboratory sessions). Constructivism has been previously used in Computer Engineering subjects [5][6][7], so it seems it is applicable to Computer Networks. Given the fact that material needed for the laboratory exercises are uncommon, lab sessions will be supported by a professor.

Practical laboratory exercises are part of students assessment, so the exercises must be evaluated. This means that, even though laboratory exercises are an important part of students learning process, their performance will be subjected to evaluation. For this reason, a formal process is established: students must do a previous work (i.e. homework) prior to entering the laboratory and, also, they must fill in a questionnaire when they are at the laboratory. The complete process and pedagogical justification are given below.

\section{A. Previous work}

Students must complete a questionnaire in preparation for the lab session that focuses on prior knowledge required and it is compulsory to show it before the lab session.

This questionnaire seeks that students understand what they are doing, not only copying one piece of information without any comprehension

\section{B. Practical exercise (lab session)}

It is difficult to follow-up the progress and performance of the students at lab as there is a rate of $12-16$ students $^{1}$ per professor in a lab session. To help the professor with the evaluation process and, more important, to help students to advance in the exercise without assistance from the professor, a questionnaire (guideline) is given to students in the lab session. This questionnaire has a series of, more or less, precise instructions on what students must do during the lab session in order to finish the tasks assigned. In addition, and there are also some questions that students must answer. These questions will be evaluated by the professor.

The stress is placed in the comprehension that students show on topics mentioned in section III and not on the numerical values obtained in this experimental process. This means that students have to analyze what they see and to combine it with the previous knowledge (previous work) in order to answer the proposed questions. To sum up, questions posed in this questionnaire try to activate students' curiosity and reflection about what they have seen.

A lab session lasts two hours and students might fill in the questionnaire individually (preferable) or in pairs.

\section{Final questionnaire (optional)}

Cooperative learning (e.g. working in pairs) is an advantage that could be taken into consideration because it allows students to work in a cooperative way. On the other hand, sometimes, resource scarce does not allow students to work

\footnotetext{
${ }^{1} 14$ students is our "official" number of students per laboratory session.

978-1-4244-4714-5/09/\$25.00 C2009 IEEE

October 18 - 21, 2009, San Antonio, TX

individually, so they are asked to work in pairs (so they can share material).

In these cases, the individual questionnaire mentioned before would not be an accurate tool to measure the performance of students because it is no longer individual. As course requirements state that each student should be evaluated individually and not in groups, another approach must be taken into consideration.

An answer to this problem might be having a final questionnaire that students should fill in individually immediately after completing the practical session.

\section{LABORATORY PREPARATION}

Materials needed to do the practical exercise are quite common in a laboratory. The following materials are required for each lab station:

- A PC with TCP/IP and an Ethernet Card (NIC).

- A digital oscilloscope (at least $100 \mathrm{MHz}$ ).

- A 10meter Cat5, solid cable. This cable must be prepared specifically for this exercise; it should have Tx and Rx pairs (i.e. Orange and Green pairs) peeled off in a way that probes from oscilloscope could be connected (see Figure 1). Both pairs must be exposed in both ends of the cable.

- Any size Cat5, solid cable. This cable should be constructed as a $10 \mathrm{~m}$ cable but, in this case, only one end pair must be exposed.

- One 2 ports (or more) 10Mbps hub; this is the hub where every student will test hub induced delay. If the hub selected is 100Mbps-capable, low speed operation should be forced on PC's Ethernet card.

Each lab station (Figure 1) operate independently of the others. In order to be able to measure hub delay, another hub must be connected to every lab station hub, so the last component needed is one n-port $10 \mathrm{Mbps}$ hub, where $\mathrm{n}$ is the number of lab stations in use. It is important to notice that hub-to-hub connection should operate at 10Mbps.

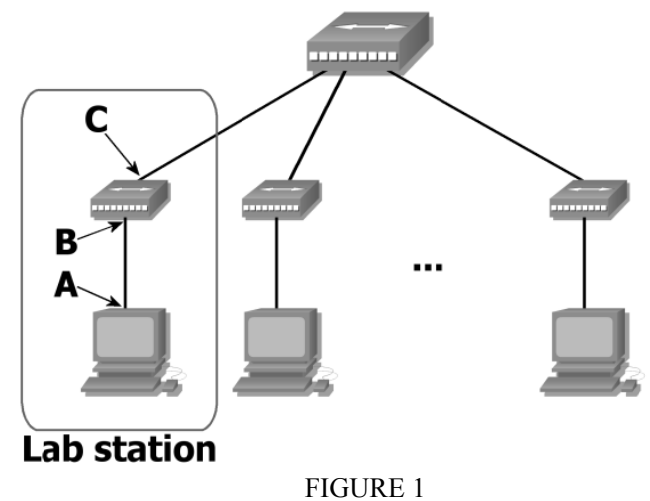

LABORATORY INFRAESTRUCTURE

The cabling used is a standard Cat5 UTP solid cable; this cable must be prepared as in Figure 2 so oscilloscope's 
probes can be easily connected (insertion points are marked with a circle in the figure).

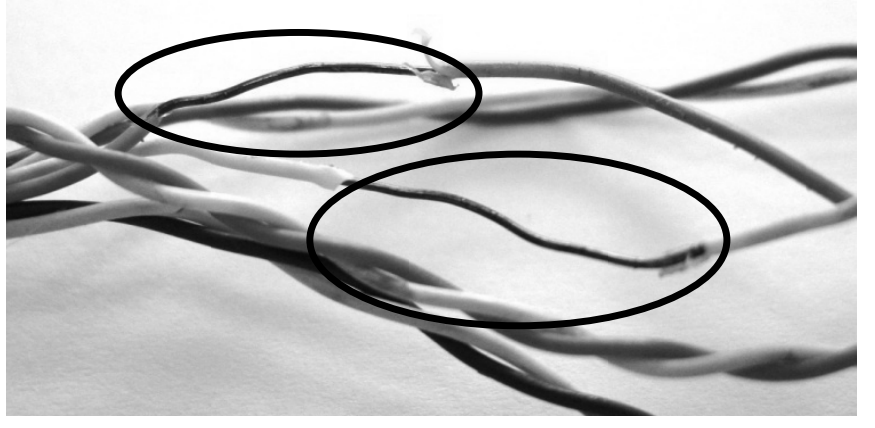

FIGURE 2

CABLE PREPARATION

Experience has shown that installing such infrastructure is possible, although not easy. The main difficulty found is the fact that every point marked $(A, B, C)$ in Figure 1 must be placed near the oscilloscope, in order to connect the probes.

\section{B. Preparing equipment}

Standard PC and communication equipment are used but, in order to have no external noise (i.e. another network traffic), an isolated network must be deployed; this means that the network devised in Figure 1 must not be connected to any external network. Also, prior to beginning the exercise, certain TCP/IP services must be disabled so every PC will only transmit frames when instructed to do so.

In order to transmit frames, it is essential that every student send the same frame. To do so, standard ping program is used with a non-existent IP address as destination (so no answer will be received). In addition, it is necessary to add a static ARP entry with a bogus MAC address; this will make the operating system to send directly the ICMP echo-request datagram, instead of ARP.

\section{Proposed Practical Exercise}

Practical exercise is divided into two parts, the first is related with frame format while the second focus on propagation delay and 5-4-3 rule. As mentioned before, a questionnaire is given to students with precise instructions on what they must do. The following paragraphs describe some of the questions that students are expected to answer, based on their initial knowledge of the matter and experimental observation.

\section{A) Frame format}

Students are asked to capture the first bits of a frame; they should select oscilloscope's resolution in such a way they can distinguish bits. In the first capture, they should capture first bits of preamble, so students are expected to obtain an image similar to Figure 3.

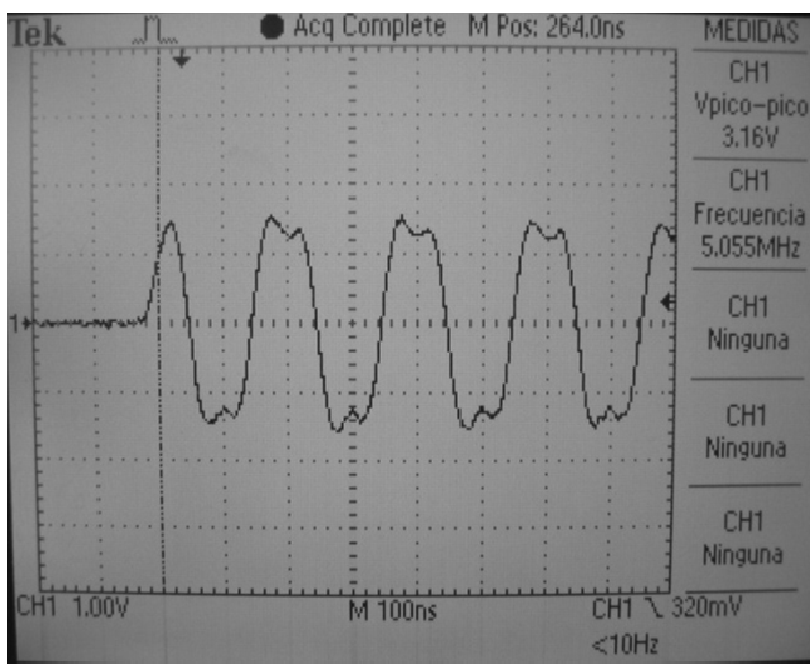

FIGURE 3

CAPTURE SHOWING FIRST BYTE OF PREAMBLE

Using this capture, and others not shown in this paper, the following questions are proposed to students (this might be included either in exercise questionnaire or in final questionnaire):

- How preamble is used at receiving side in order to synchronize? Students are expected to recognize this waveform as a Manchester encoding, because they know preamble is a sequence of 10101010. Also they should infer that this is a $5 \mathrm{MHz}$ square waveform whose transitions always identify a bit and that means receiver knows where zeroes and ones are without the need of an external clock (auto synchronization).

- Why is so easy to localize Starting Frame Delimiter (SFD) in the frame? Is there any problem if some bits of preamble are lost (in order to localize SFD)? Students know (previous knowledge) that SFD is a single sequence of 10101011 using a Manchester encoding. Students should infer that the last bits (11) will cause waveform to change twice (in the same bitperiod) so, even if some bits of preamble are lost, it is very easy to identify the SFD after the preamble.

- Specify the first three bytes of destination address in canonical notation: Since they know IEEE802.3's frame format, they should be able to decode the destination address. They should also be aware of the differences between canonical notation and bit transmission ordering.

- Measure frame length and compare it with minimum frame length: This allows students to see that if data to be transmitted are less than the minimum specified by IEEE802.3, frame length will always be 512bits (data field is filled by padding bytes, if necessary). Students will use ICMP for that because it is possible to send ICMP messages with different sizes. 
B) Delays and 5-4-3 rule

In the first part only one channel in the oscilloscope is required, in the second part students require two probes in order to measure signal attenuation, delay and hub delay.

The following questions are proposed (may be included in either questionnaire):

- Measure signal attenuation and delay (cable): Students are asked to measure these parameters within a $10 \mathrm{~m}$ cable (marked as A and B on Figure 1). They should be able to calculate signal propagation speed. Also, they are asked to obtain signal attenuation.

- Measure signal attenuation and delay (hub): Students are required to do the same calculation as before but with probes connected to the station's hub, one probe connected to the input port and other probe connected to the output port (marked as B and C on Figure 1). Students should notice that hub regenerates signal (signal is amplified) and, more important, it introduces a delay; in order to make them comprehend that this delay is relevant, they are asked to translate the delay in a hub to a equivalent distance. Figure 4 shows hub delay as it is captured in the oscilloscope. It is also useful to show students that different hubs introduce different delays. This should allow them to understand why limits like 54-3 rule are designed to work in worst-case scenario and as a consequence, exceeding that limits do not mean the network will cease functioning unconditionally (it depends on the unique characteristics of the network).

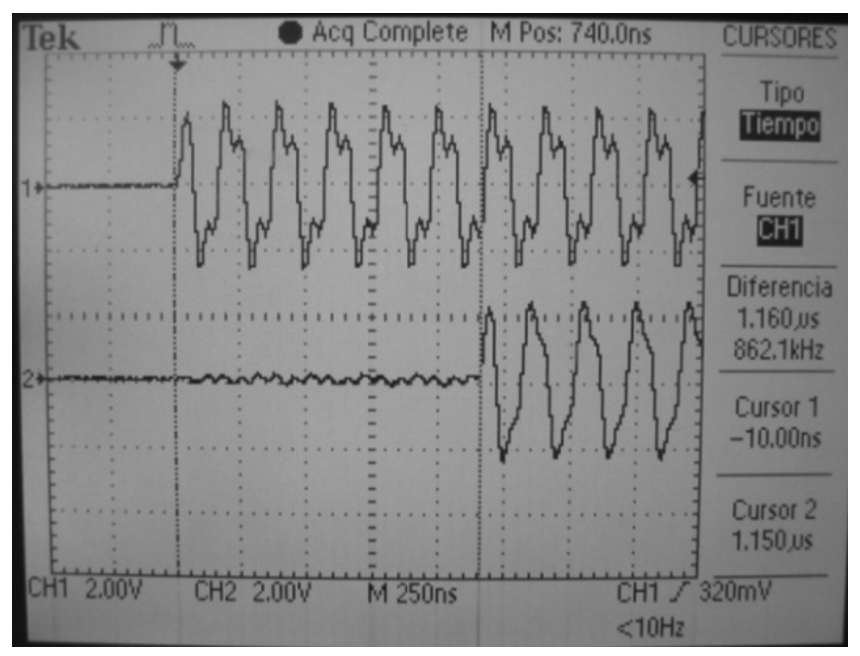

FIGURE 4

HUB DELAY $(1.16 \mu \mathrm{s})$

- Determine minimum frame length for CSMA/CD to operate properly in THIS network: Once students have realized that the unique network deployed in laboratory has a unique Round Trip Delay (RTD), they are asked to calculate the minimum frame length in this network. This should allow students to comprehend
CSMA/CD behavior and its relation with 5-4-3 rule by applying it to any specific scenario. In our hands-on experience students did not complete this question due to lack of time.

\section{C) Additional questions}

Previous questions were part of questionnaires presented to students, but there are still some topics that might be added:

- Measure IFG length: In order to have an IFG of 96 bits (minimum allowed), a 10/100 Mbps switch must be used, so PC transmits frames at $100 \mathrm{Mbps}$ and probe is connected to a $10 \mathrm{Mbps}$ hub connected to the switch.

- Different switch operating modes: Switches may operate in two different commutation modes (store and forward, and fast forwarding); students will observe differences between them and analyze why fast forwarding mode introduces less latency than store and forward.

\section{HANDS-ON EXPERIENCE}

In order to have a hands-on experience, a group of students were selected; they were students enrolled in a noncompulsory subject at fifth year which focus on advanced networking topics. They were, therefore, a group of students with certain motivation for computer networks.

\section{A. Observed experience}

The first problem observed was trying to install laboratory infrastructure; it is highly recommended that some sort of external structured wiring be available in laboratory and enough space around the lab station.

Secondly, it seems necessary to give very specific instructions on how to use oscilloscope in order to capture a frame. Proposed questionnaire did not give much instructions and that meant that the professor in charge had to explain students "one-by-one" how to use the oscilloscope (although students have used them in the first years of the degree).

This kind of practical exercise requires constant attention from the professor so optimal group size could be 12-students and, in any case, no more than 14.

\section{B. Students evaluation}

Students opinion about the exercise is essential. In this case, a single group of 16 students was formed. After completing the practical exercises an evaluation questionnaire was given out to students, so feedback about the exercise could be obtained. The questionnaire asked students to evaluate the practical exercises in five topics: Manchester codification, synchronization mechanisms, canonical notation and bit transmission ordering, attenuation and delay (cable), and delay in a hub. For every topic, two questions were made: "My knowledge of the topic BEFORE the practical exercise is $(0-10)$ " (Q1) and "I consider that the practical exercise has helped me in understanding the topic (0-10)" (Q2). 0 means "no knowledge" for Q1 and "no help" for Q2. The data collected is given in Table 1.

October 18 - 21, 2009, San Antonio, TX 
Session M2E

TABLE 1

RESULTS

\begin{tabular}{|c|c|c|c|c|}
\hline Topic & Q1 mean & $\begin{array}{c}\text { Q1 std } \\
\text { deviation }\end{array}$ & Q2 mean & $\begin{array}{c}\text { Q2 std } \\
\text { deviation }\end{array}$ \\
\hline $\begin{array}{c}\text { Manchester } \\
\text { codification }\end{array}$ & 7,35 & 1,34 & 8,43 & 1,55 \\
\hline Synchronization & 6,36 & 2,31 & 8,5 & 1,64 \\
\hline $\begin{array}{c}\text { Transmission } \\
\text { order }\end{array}$ & 6,79 & 1,86 & 7,93 & 1,49 \\
\hline $\begin{array}{c}\text { Attenuation and } \\
\text { delay }\end{array}$ & 6,14 & 2,23 & 8,79 & 1,32 \\
\hline Hub delay & 5,29 & 2,02 & 8,23 & 1,67 \\
\hline
\end{tabular}

These results show that the topics studied in the practical exercises are, in fact, tough to students. The impression students have about their own knowledge shows that the average thinks that something could be done. However, results also show that standard deviation is a little high; this means previous knowledge is not homogeneous to every students. Anyway, it seems that Manchester encoding, according to students is not a difficult topic but it does not mean that this topic should be removed from the questionnaire, because it is necessary for the understanding of other topics.

On the other hand, the students perceive the exercise as useful; on average they give a score of 8 out of 10 when evaluating "the exercise has helped me to understand better this topic"; in this case standard deviation of the results appears to be better (less dispersion) than in the first question.

So, it could be concluded that students, on average, have a high perception of exercise usefulness.

\section{Students performance}

The proposed methodology used states the convenience of a practical exercise questionnaire which activates students' curiosity and comprehension of the topics studied.

Students were asked to answer these questions individually so it is a good reference of students' performance. The average grade was 7,54 out of 8 , being the lowest grade 6,5 and the mode 8 . These results seem to confirm usefulness of the practical exercise proposed for the students.

\section{CONCLUSIONS}

Physical and MAC related topics in the IEEE 802.3 specification are usually difficult for Computer Network students. There is also a historical lack of practical laboratory exercises because they usually focus on higherlever layers.

On the other hand, making students to learn in a constructive way is especially useful in this situation. Students learn these problematic topics at lectures and use practical exercises as a continuation of the lecture. The practical exercise proposed, for this reason, is not something independent of lectures; on the contrary, practical exercise is a continuation of lectures, and students acquire a better comprehension of these topics by applying knowledge previously learned at lectures at laboratory.

Practical exercise proposed is composed of two parts, the first (previous work) is some sort of homework that student must work with it before entering the laboratory. This previous work is an important part of the exercise, because it helps students to revise concepts that will be needed at laboratory. The second part is a questionnaire that students fill in during lab session; this is the most important part of the exercise because it allows students to construct a new knowledge using previously explained theory and practical observation.

Experience has shown that students evaluate the practical exercise as useful as it seems in every topic covered in the practical exercise; this experience has help students to improve their understanding of them.

\section{ACKNOWLEDGMENT}

This paper has been supported by the "I Plan Propio de Docencia" funded by the University of Seville, Spain and by the research project TOMARES (TEC2006-08430) Multimedia Operatives Techniques applied to Supply Electric Networks- funded by the Ministry of Education and Science of Spain.

\section{REFERENCES}

[1] Behrouz A. Forouzan. "Data Communications and Networking", 4th ed, Mac-Graw Hill, 2007.

[2] William Stallings. "Data and Computer Communications", 8th ed, Prentice Hall, 2007.

[3] Charles E. Spurgeon, "Ethernet: The Definitive Guide", 1st ed, O'Reilly, 2000

[4] IEEE Standard for Information technology, Local and metropolitan area networks - Part 3: Carrier sense multiple access with collision detection (CSMA/CD) access method and physical layer specifications, IEEE standard 802.3, 2005.

[5] M. Grigoriadou, E. Kanidis and A. Gogoulou. "A web-based educational environment for teaching the computer cache memory", IEEE trans. on Education, vol 49, pp 147-156, Feb 2006.

[6] L. Moreno, C. Gonzalez, I. Castilla, E.J. Gonzalez, ; J. Sigut. "Use of Constructivism and Collaborative Teaching in an ILP Processors Course", IEEE trans. on Education, vol 50, pp. 101-111, May 2007.

[7] S. Hadjerrouit. "Learner-centered web-based instruction in software engineering", IEEE trans. on Education, vol. 48, pp. 99-104, Feb 2005 